\title{
Efectividad de dos Formulaciones de Clethodim en el Control de Gramíneas y su Antagonismo con Bentazon
}

\author{
Elvis Erbuins Espinoza Valdés , Abelino Pitty² y Rogelio Trabanino
}

Resumen: Los objetivos fueron evaluar la eficacia de las formulaciones Icasso 24 EC y Arrow 12 EC del herbicida Clethodim y determinar el antagonismo de ambas formulaciones con Bentazon. En dos experimentos se evaluó el control de malezas gramíneas y la fitotoxicidad al frijol, y en otro se evaluó el antagonismo al mezclar en el tanque el Clethodim (120 g de ingrediente activo por hectárea) con Bentazon (1,440 g de ingrediente activo por hectárea). En todos los experimentos se aplicó con una bomba de mochila de acero inoxidable modelo T presurizada con $\mathrm{CO}_{2}$ a una presión de 35 psi, un aguilón de $2 \mathrm{~m}$ de ancho con cuatro boquillas de abanico plano. En todos los ensayos se usó un diseño experimental de bloques completamente al azar y ninguno de los resultados de los testigos sin aplicación de herbicida, se usó en el análisis estadístico. La separación de medias fue con la prueba Duncan al $5 \%$. En el primer ensayo, a las 2 semanas después de la aplicación, la formulación Icasso 24 EC tuvo $86 \%$ de control y para Arrow 12 EC fue $73 \%$, ambas se aplicaron a $96 \mathrm{~g}$ del ingrediente activo por hectárea. En el segundo ensayo a las 3 semanas después de la aplicación, usando $96 \mathrm{~g}$ de ingrediente activo por hectárea, el control con Icasso 24 EC fue 40\% y con Arrow 12 EC 33\%, al aumentar la dosis a $120 \mathrm{~g}$ el control fue $45 \%$ con Icasso $24 \mathrm{EC} \mathrm{y} 36 \%$ Arrow $12 \mathrm{EC}$. El control fue mayor con Icasso $24 \mathrm{EC}$, que con Arrow $12 \mathrm{EC}$, por lo tanto, se atribuye la diferencia en el control de malezas a la formulación del herbicida, ya que los fabricantes añaden ingredientes inertes diferentes y algunos aumentan la eficacia del herbicida. En ninguna evaluación las formulaciones causaron fitotoxicidad al frijol. Esto indica que ninguna de las formulaciones ni los ingredientes inertes dañan al frijol. La evaluación del antagonismo fue un factorial y hubo diferencia estadística en el control entre las formulaciones y cuando las formulaciones se aplicaron solas o mezcladas con Bentazon. En ninguna evaluación hubo interacción, lo que indica que los factores (formulación y la mezcla con Bentazon) actúan independientemente. A las 3 semanas después de la aplicación, al mezclar Bentazon con Icasso $24 \mathrm{EC}$ el control se redujo de 74 a $25 \%$, una reducción de $66 \%$. Al mezclar Bentazon con Arrow 12 EC el control se redujo de 60 a 11\%, una reducción de $82 \%$. El Bentazon es antagonista a las dos formulaciones, pero hubo mayor antagonismo o reducción en el control con Arrow 12 EC que con Icasso $24 \mathrm{EC}$.

Palabras clave: Herbicida de contacto, herbicida selectivo, ingrediente activo, ingrediente inerte.

\section{Efficacy of two Formulations of Clethodim on Grass Control and its Antagonism with Bentazon}

\begin{abstract}
The objectives were to evaluate the efficacy of Icasso 24 EC and Arrow 12 EC, two commercial formulations of the herbicide Clethodim, and to determine the antagonism between Clethodim and Bentazone. In two trials, grass weed control and phytotoxicity to dry bean were evaluated; in another trial, the antagonism was evaluated, by mixing the two formulations of Clethodim (120 g of active ingredient per hectare) in the tank with Bentazone (1,440 $\mathrm{g}$ of active ingredient per hectare). All applications were made with a stainless steel backpack sprayer, model $\mathrm{T}$ pressurized with $\mathrm{CO}_{2}$ at $35 \mathrm{psi}$, a $2 \mathrm{~m}$ wide boom, with four flat fan nozzles spaced $50 \mathrm{~cm}$ between them. A completely randomized blocks design was used. In all the trials, data from the controls without herbicide applications were not used in the statistical analysis. The mean separation of results was established with Duncan test at 5\%. In the first trial, at 2 weeks after application, the formulation Icasso 24 EC had $86 \%$ control and Arrow $12 \mathrm{EC}$ had $73 \%$, both were applied at $96 \mathrm{~g}$ of active ingredient per hectare. In the second first trial, at 3 weeks after application, using $96 \mathrm{~g}$ of active ingredient per hectare, the control with Icasso 24 EC was $40 \%$ and with Arrow $12 \mathrm{EC} 33 \%$, increasing the rate to $120 \mathrm{~g}$ of active ingredient per hectare weed control was 45\% with Icasso $24 \mathrm{EC}$ and 36\% with Arrow $12 \mathrm{EC}$. The control was better with Icasso $24 \mathrm{EC}$, than with Arrow 12 $\mathrm{EC}$, therefore, the difference in weed control is attributed to the formulation of the herbicides, since
\end{abstract}

\footnotetext{
${ }^{1}$ Consultor independiente, Dolega, Chiriquí, Panamá. Correo electrónico elvis1800@hotmail.com

2 Escuela Agrícola Panamericana, Zamorano, Honduras. Correo electrónico apitty@zamorano.edu

3

Escuela Agrícola Panamericana, Zamorano, Honduras. Correo electrónico rtrabanino@zamorano.edu
}

DOI: 10.5377/ceiba.v53i1.2016 
manufacturers add different inert ingredients that enhance the efficacy of the herbicide. No phytotoxicity to dry bean was observed in any of the experiments. This indicates that none of the formulations nor inert ingredients caused damage to the dry bean. A factorial design was used for the antagonism evaluation, statistical differences were found between formulations and when the formulations were applied alone or mixed with Bentazone. In all evaluations there was no interaction, which indicates that the factors (formulation and mixing with Bentazone) act independently. At 3 weeks after the application, of the mixture of Icasso 24 EC with Bentazone, control was reduced from 74 to $25 \%$, a reduction of $66 \%$. When mixing Bentazone with $12 \mathrm{EC}$ Arrow the control was reduced from 60 to $11 \%$, a reduction of $82 \%$. Based on these results it is concluded that Bentazon is antagonistic to both formulations of Clethodim, and there is a greater antagonist or reduction in the control with Arrow $12 \mathrm{EC}$ than with Icasso $24 \mathrm{EC}$.

Keywords: Active ingredient, contact herbicide, inert ingredient, selective herbicide.

\section{Introducción}

El Clethodim (2-[(1E)-1-[[[(2E)-3-chloro-2 propenyl]oxy]imino] propyl]-5-[2-(ethylthio) propyl]-3hydroxy-2-cyclohexen-1-one) es un herbicida selectivo de posemergencia. Actúa inhibiendo la acción de la acetil coenzima A carboxilasa, la enzima comprometida en la catalización de nuevos ácidos grasos (WSSA 2007). El crecimiento cesa en los tejidos jóvenes y en crecimiento; una clorosis y luego una necrosis se desarrollan entre la semana uno y tres después de la aplicación (WSSA 2007). La translocación del Clethodim, presumiblemente, es similar al Sethoxydim, se mueve principalmente en el simplasto (incluyendo el floema) y se acumula en los puntos de crecimiento (WSSA 2007).

Clethodim es fabricado bajo varios nombres comerciales, algunos son: Select ${ }^{\circledR}$, Prism $^{\circledR}$, Arrow $^{\circledR}$, Envoy $^{\circledR}$, Segard ${ }^{\circledR}$, Ospray ${ }^{\circledR} \quad$ (WSSA 2007), Crop\$mart ${ }^{\mathrm{TM}}$, Diler, Gramadal, Clethodim y Grass Out Max. El ingrediente activo, Clethodim, es el mismo en todas las formulaciones, pero los ingredientes inertes pueden ser diferentes y generalmente los ingredientes inertes y las cantidades añadidas son desconocidos.

La eficacia de los herbicidas es influenciada por los adyuvantes en la formulación, así como la especie de maleza, la calidad del agua y las condiciones climáticas (Hatzios y Penner 1985). Las diferencias en los ingredientes inertes en las formulaciones pueden afectar la deposición y retención en la hoja, la absorción (Dodds et al. 2007) y translocación en la planta (Wanamarta y Penner 1989). Además, pueden disminuir la exposición del Clethodim a la luz ultravioleta (Nandula et al. 2007) y aumentar el control ya que la luz ultravioleta degrada al Clethodim (Ahrens 1994).
Los ingredientes inertes, además de aumentar la eficacia (Culpepper et al. 1999a), la facilidad de manejar la formulación y la vida de anaquel del ingrediente activo, también pueden causar fitotoxicidad en el cultivo en el cual se aplica. Por lo tanto, se deben evaluar las nuevas formulaciones para comparar su eficacia y daño a los cultivos con las formulaciones ya en el mercado.

La adición de adyuvante a cualquier formulación de Clethodim aumenta la absorción del Clethodim y el control de la maleza Cynodon dactylon. Aplicándolo con adyuvantes es mejor, ya que mejora la absorción y translocación del herbicida en la planta, en comparación de aplicarlo sin adyuvante (Nandula et al. 2007). Se presume que el comportamiento sea similar en la maleza estrella africana (Cynodon nlemfuensis), ya que pertenecen a la misma familia (Poaceae).

En los sistemas de producción de cultivos convencionales es habitual mezclar varios herbicidas en el tanque para reducir los costos de aplicación. Sin embargo, en algunos casos causa reacciones antagónicas que reducen el control de malezas (Holshouser y Coble 1990, Burke et al. 2002).

Antagonismo es el nombre dado a la interacción negativa entre dos o más compuestos (Kruse et al. 2006). En general, cuando la mezcla de productos tiene un efecto menor que la de los productos individuales, se dice que existe antagonismo (De Prado y Osuna 1999). Hay herbicidas que con otros productos son incompatibles y su mezcla no es aconsejable porque existe una acción imprevisible o que sus mecanismos de acción interfieran entre sí.

El control de malezas gramíneas anuales y 
perennes se reduce debido a la mezcla de Sethoxydim con herbicidas para el control de hojas anchas, tales como Bentazon o Acifluorfen (Wanamarta et al. 1989, Holshouser y Coble 1990). Sethoxydim y Clethodim son herbicidas de la misma familia (Ciclohexanodionas), por lo tanto se esperaría que tengan un comportamiento similar. Se ha reportado que Bentazon reduce la absorción foliar del Sethoxydim en la maleza gramínea Eleusine indica (Rhodes y Coble 1984, Holshouser y Coble 1990).

El Bentazon (3-(1-methylethyl)-1H-2,1,3benzothiadiazin-4(3H)-one 2,2-dioxide) es un herbicida selectivo de posemergencia. Actúa Inhibiendo la fotosíntesis en el fotosistema II, ocurre una reducción de la plastoquinona por la proteína $D_{1}$ en la membrana del tilacoide. Este herbicida se enlaza a la proteína $D_{1}$, con lo que se obstruye el enlace de la plastoquinona. Al inhibirse el enlace, el proceso de transferencia fotosintética de electrones es interrumpido y por lo tanto la síntesis de ATP y NADPH en el cloroplasto es afectada. Esto da como resultado una incapacidad para la fijación de $\mathrm{CO}_{2}$, necesaria para la producción de los carbohidratos indispensables para la sobrevivencia de la planta (WSSA 2007). Los síntomas de daños se manifiestan como clorosis, disecación foliar y necrosis que se desarrollan entre los 3 a 5 días después de la aplicación (WSSA 2007). Las plantas lo absorben a través de sus hojas y la translocación es basipetal a varios tejidos (WSSA 2007).

Al mezclar en el tanque Bentazon con Sethoxydim, la absorción del Sethoxydim por las hojas es menor (Holshouser y Coble 1990), inhibiéndose severamente el control de malezas del Sethoxydim debido al sodio presente en la molécula del Bentazon. Reemplazando el sodio del Bentazon por las sales de amonio se previene el antagonismo a la absorción del Sethoxydim (Wanamarta et al. 1989). En este caso las sales de amonio (sulfato, fosfato o nitrato de amonio) se unen al Sethoxydim y se elimina el efecto antagónico causado por el sodio del Bentazon. Esto sucede gracias a la inhibición por las sales de amonio a los iones de sodio $\mathrm{Na}^{+}$(Wanamarta et al. 1989).

Los objetivos fueron determinar el control de malezas gramíneas con el ingrediente activo Clethodim, usando las formulaciones Icasso $24 \mathrm{EC}^{4} \mathrm{y}$

\footnotetext{
${ }^{4}$ Icasso fabricado por Rotam en febrero del 2012, lote número 20120229001
}

Arrow $12 \mathrm{EC}^{5}$; determinar si hay fitotoxicidad al frijol con las dos formulaciones y si existe antagonismo al mezclar en el tanque las formulaciones de Clethodim con Bentazon.

\section{Materiales y Métodos}

Evaluación de la eficacia de las formulaciones. El frijol variedad De Orho se sembró el 2 de marzo de 2013, se aplicó el insecticida Imidacloprid - Thiodicarb (Blindage $60 \mathrm{FS}$ ) a razón de $4 \mathrm{ml}$ del producto comercial por kilogramo de semilla. Se fertilizó con DAP 18-46-0 a $159 \mathrm{~kg} / \mathrm{ha}(3.50 \mathrm{qq} / \mathrm{ha})$. La distancia entre surcos fue de $50 \mathrm{~cm}$ y se colocaron 10 semillas por metro lineal (densidad de 200,000 plantas por hectárea). Los lotes fueron irrigados con un pivote central según la necesidad del cultivo.

Se marcaron los lotes de $10 \times 5 \mathrm{~m}$ en un arreglo de bloques totalmente aleatorizados con cuatro repeticiones. Ninguno de los testigos sin aplicación de herbicida se usó en el análisis estadístico. La separación de medias se realizó con la prueba Duncan al $5 \%$. Los tratamientos fueron:

- Icasso 24 EC (Clethodim) $400 \mathrm{ml} / \mathrm{ha}$, equivalente a $96 \mathrm{~g}$ de ingrediente activo

- Icasso 24 EC (Clethodim) $500 \mathrm{ml} / \mathrm{ha}$, equivalente a $120 \mathrm{~g}$ de ingrediente activo

- Arrow 12 EC (Clethodim) $800 \mathrm{ml} / \mathrm{ha}$, equivalente a $96 \mathrm{~g}$ de ingrediente activo

- Testigo absoluto sin control de malezas

- Testigo desyerbado con azadón (para determinar fitotoxicidad).

Se desmalezaron los lotes testigos con azadón a los 20 días después de la siembra; 5 días después se aplicaron todos los herbicidas en una mezcla con 233 L/ha. Se aplicó con una bomba de mochila modelo $T$, de acero inoxidable 6 , presurizada con $\mathrm{CO}_{2}$ a una presión de 35 psi, un aguilón de $2 \mathrm{~m}$ de ancho con cuatro boquillas tipo abanico plano 8003 separadas $50 \mathrm{~cm}$ entre ellas.

\footnotetext{
${ }^{5}$ Arrow fabricado y formulado por Proficol Andina, Colombia, en noviembre de 2011 , lote número PF 11111174

${ }^{6}$ Mochila modelo $\mathrm{T}$, equipada con contenedor de acero inoxidable de $11.35 \mathrm{~L}$, cilindro de aluminio de $\mathrm{CO}_{2}$ de 2.27 kg, regulador de presión y aguilón para cuatro boquillas. Fabricado por Bellspray, Inc.; P.O. Box 269, Opelousas, LA 70571-0269, Estados Unidos.
} 
A todos los tratamientos con herbicidas se le agregó Bentazon (Basagran $48 \mathrm{SL}^{7}$ ) a $3.0 \mathrm{~L} / \mathrm{ha}$ equivalente a $1,440 \mathrm{~g}$ de ingrediente activo por hectárea; además se añadió el surfactante Adsee ${ }^{\circledR}$ $775^{8}$ a $0.25 \% \mathrm{v} / \mathrm{v}$. Debido a que la flora de malezas está compuesta por gramíneas y hojas anchas, se mezcló el_Bentazón (control de hojas anchas) con el Clethodim (control de gramíneas), que es una aplicación estándar en el frijol para controlar los dos tipos de malezas.

El control de malezas y el daño al cultivo se determinó visualmente siguiendo la metodología del Australian Weeds Committee (1979). Los daños se evaluaron en porcentaje y no hubo transformación al arcoseno porque los datos tenían una distribución normal, no había valores extremos de 0 ni $100 \%$. El testigo absoluto ( $0 \%$ de control) se usó para comparar el control de malezas con los lotes aplicados. El testigo desyerbado con azadón ( $0 \%$ de fitotoxicidad) se usó para comparar la posible fitotoxicidad al frijol causada por las formulaciones.

Para evitar el efecto del borde, en cada lote se eliminaron los dos surcos exteriores y $1.0 \mathrm{~m}$ al inicio y al final de los Ites. Se evaluó el control de malezas y el daño al cultivo 1 y 2 semanas después de la aplicación. Los porcentajes del control se tomaron con dos evaluadores y se tomó el promedio de los dos evaluadores como el control de maleza.

Evaluación de las dos formulaciones de Clethodim utilizando dos dosis. El experimento se estableció en un lote de producción llamado El Pivote perteneciente a la Escuela Agrícola Panamericana, Zamorano, Honduras. El 2 de mayo de 2013, el frijol se sembró con sembradora en camas levantadas de $30 \mathrm{~cm}$ de alto ya que se acercaba la época lluviosa. La distancia del centro de una cama a otra era de $1.50 \mathrm{~m}$ y la distancia entre los surcos de frijol en la cama era de $50 \mathrm{~cm}$. Los lotes eran de $10 \times 6 \mathrm{~m}$. El 11 de mayo de 2013, los testigos se desmalezaron con azadón. Ese mismo día, se aplicaron los herbicidas, los tratamientos fueron:

- Icasso 24 EC (Clethodim) $400 \mathrm{ml} / \mathrm{ha}$, equivalente a $96 \mathrm{~g}$ de ingrediente activo

- Icasso 24 EC (Clethodim) $500 \mathrm{ml} / \mathrm{ha}$, equivalente a $120 \mathrm{~g}$ de ingrediente activo

\footnotetext{
${ }^{7}$ Basagran fabricado y formulado por BASF SE, BASF Societas Europaea, Alemania, lote número 1444074700.

${ }^{8}$ Adsse fabricado por West Trade Guatemala.
}

- Arrow 12 EC (Clethodim) $800 \mathrm{ml} / \mathrm{ha}$, equivalente a $96 \mathrm{~g}$ de ingrediente activo

- Arrow 12 EC (Clethodim) $1000 \mathrm{ml} / \mathrm{ha}$, equivalente a $120 \mathrm{~g}$ de ingrediente activo

- Testigo absoluto sin control de malezas

- Testigo desyerbado con azadón (para determinar fitotoxicidad).

La aplicación fue igual que en el experimento anterior (equipo, boquillas, presión), pero la cantidad de agua fue menor, se utilizó una mezcla de $217 \mathrm{~L} / \mathrm{ha}$. La toma de datos fue realizada en las semanas 1, 2 y 3 después de la aplicación de los herbicidas y fue similar a la del experimento anterior.

A todos los tratamientos con herbicidas se le agregó Bentazon a $3.0 \mathrm{~L} / \mathrm{ha}$ equivalente a $1,440 \mathrm{~g}$ de ingrediente activo por hectárea; además se añadió el surfactante Adsee ${ }^{\circledR} 775$ a $0.25 \%$ v/v. Debido a que la flora de malezas está compuesta por gramíneas y hojas anchas, se mezcló el Bentazón (control de hojas anchas) con el Clethodim (control de gramíneas), que es una aplicación estándar en el frijol para controlar los dos tipos de malezas.

La evaluación del control de malezas fue similar a la que se hizo en el primer experimento. Se usó un diseño de bloques totalmente al azar y ninguno de los datos de los testigos sin aplicación de herbicida se usó en el análisis estadístico. La separación de medias fue con la prueba Duncan al $5 \%$.

Evaluación del antagonismo entre Clethodim y Bentazon. El experimento se estableció el 6 de junio de 2013, se usó un lote en el cual no existía cultivo, la maleza predominante era el pasto estrella (Cynodon nlemfuensis).

Se marcaron parcelas de $8 \times 4 \mathrm{~m}$ en un arreglo de bloques totalmente al azar con cuatro repeticiones. Los tratamientos fueron:

- Icasso 24 EC (Clethodim) $500 \mathrm{ml} / \mathrm{ha}$, equivalente a $120 \mathrm{~g} / \mathrm{ha}$ de ingrediente activo

- Arrow 12 EC (Clethodim) $1000 \mathrm{ml} / \mathrm{ha}$, equivalente a $120 \mathrm{~g} / \mathrm{ha}$ de ingrediente activo

- Icasso 24 EC (Clethodim) $500 \mathrm{ml} / \mathrm{ha}$, equivalente a $120 \mathrm{~g} / \mathrm{ha}$ de ingrediente activo, combinado con Basagran $48 \mathrm{SL}$ (Bentazon) $3000 \mathrm{ml} / \mathrm{ha}$ equivalente a $1440 \mathrm{~g} / \mathrm{ha}$ de ingrediente activo

- Arrow 12 EC (Clethodim) $1000 \mathrm{ml} / \mathrm{ha}$, equivalente a $120 \mathrm{~g} / \mathrm{ha}$ de ingrediente activo, combinado con Basagran $48 \mathrm{SL}$ (Bentazon) 
Espinoza Valdés et al.: Efectividad de dos Formulaciones de Clethodim en el Control de Gramíneas

$3000 \mathrm{ml} / \mathrm{ha}$ equivalente a $1440 \mathrm{~g} / \mathrm{ha}$ de ingrediente activo.

- Testigo absoluto sin control de malezas con herbicidas, ni desmalezado a mano.

La aplicación de los herbicidas se realizó igual que los experimentos anteriores (equipo, boquillas y presión). La cantidad de agua fue de 275 L/ha y a todos los tratamientos se añadió el surfactante Adsee $^{\circledR} 775$ a $0.25 \% \mathrm{v} / \mathrm{v}$. La toma de datos también fue similar que en los dos experimentos anteriores, se evaluó el control en la semana 1, 2 y 3 después de aplicar los herbicidas.

El diseño fue bloques completamente al azar y se analizó como un arreglo factorial de $2 \times 2$. El testigo no se usó en el análisis estadístico. La separación de medias fue con la prueba Duncan al $5 \%$.

\section{Resultados y Discusión}

Evaluación de la eficacia de las formulaciones. Las malezas gramíneas predominantes eran Eleusine indica, Digitaria spp., Chloris virgata, Chloris radiata y Sorghum halepense. En la primera evaluación, una semana después de la aplicación, el control de gramíneas fue similar con las dos dosis de la formulación Icasso $24 \mathrm{EC}$, sin embargo, el control con Arrow 12 EC fue menor que con las dos dosis de Icasso 24 EC (Cuadro 1). La misma respuesta ocurrió a las 2 semanas, con Icasso 24 EC, en sus dos dosis, el control fue estadísticamente mayor que con Arrow 12 EC (Cuadro 1).

Cuando la cantidad de ingrediente activo aplicado fue la misma en las formulaciones Icasso 24 EC y Arrow 12 EC (96 g/ha de ingrediente activo), el control fue mayor con lcasso $24 \mathrm{EC}$, por lo tanto, se atribuye la diferencia en el control de malezas a la formulación ya que los fabricantes añaden ingredientes inertes diferentes y algunos de los ingredientes inertes aumentan la eficacia del herbicida (Culpepper et al. 1999a). La eficacia puede aumentar por una mayor absorción del herbicida (Dodds et al. 2007), por un mayor contacto con la hoja o menos lavado por la lluvia (Culpepper et al. 1999b).

No se observó fitotoxicidad al frijol causada por las formulaciones. Esto indica que ninguna de las formulaciones del ingrediente activo daña al frijol, y tampoco los ingredientes inertes que tiene el Icasso 24 EC o el Arrow 12 EC.
Evaluación de las dos formulaciones de Clethodim utilizando dos dosis. Las malezas gramíneas eran Eleusine indica, Digitaria spp., Chloris virgata, Chloris radiata, Sorghum halepense y Rottboelia cochinchinensis. El control a la primera semana después de la aplicación fue errático, el mayor control (43\%) fue con Icasso 24 EC a $96 \mathrm{~g} / \mathrm{ha}$ de ingrediente activo y fue mejor que el lcasso 24 EC (36\%) con la dosis de $120 \mathrm{~g} / \mathrm{ha}$ de ingrediente activo; con Arrow 12 EC fue similar (Cuadro 2). Al final de la segunda semana, el control fue mayor con Icasso 24 EC en las dos dosis (60\%) que las dos dosis del Arrow 12 EC (50 y 49\%). A la tercera semana, el mayor control de todos los tratamientos fue con Icasso $24 \mathrm{EC}$ a $120 \mathrm{~g} / \mathrm{ha}$ de ingrediente activo (45\%). El control más bajo fue con Arrow 12 EC a $96 \mathrm{~g} / \mathrm{ha}$ de ingrediente activo y de $120 \mathrm{~g} / \mathrm{ha}$ de ingrediente activo, 33 y $36 \%$, respectivamente (Cuadro 2 ).

En todos los tratamientos, el control a la segunda semana fue mayor que el de la primera semana, pero bajó de la segunda a la tercera semana ya que las malezas se recuperaron del efecto del herbicida y empezaron a crecer nuevamente. Una posible explicación al control bajo fue que había un antagonismo al mezclar en el tanque el Clethodim con Bentazon (Basagran). Se ha reportado una reducción en el control de malezas cuando se mezcla Clethodim con los herbicidas Acifluorfen (Blazer), Bromoxynil (Culpepper et al. 1999a, Culpepper et al. 1999b) o Basagran (Bentazon) (WSSA 2007, Kidd y James 1991) y aún con otros herbicidas (Burke y Wilcut 2003, Burke et al. 2002).

Se observó un estrés por falta de agua, posiblemente porque se sembró en camas altas, por lo tanto era más difícil proporcionar suficiente agua al cultivo ya que durante el riego el agua escurría de la cama al fondo del surco. En el experimento anterior no se sembró en camas y no se observó estrés por falta de agua. Posiblemente, el estrés de las malezas por falta de agua en este experimento favoreció la manifestación de un posible antagonismo, ya que se ha reportado más antagonismo con los herbicidas Fluazifop y Acifluorfen cuando hay estrés por sequía (Godley y Kitchen 1986).

No se observó daño al frijol con ninguna de las formulaciones del herbicida. Esto confirma que el ingrediente activo no daña al frijol, tampoco los ingredientes inertes. 
Cuadro 1. Porcentaje del control de malezas gramíneas con dos formulaciones del herbicida Clethodim".

\begin{tabular}{lcccc}
\hline $\begin{array}{c}\text { Nombre comercial y } \\
\text { formulación }\end{array}$ & $\begin{array}{c}\text { Producto } \\
\text { comercial (ml/ha) }\end{array}$ & $\begin{array}{c}\text { Ingrediente activo } \\
(\mathbf{g} / \mathbf{h a})\end{array}$ & \multicolumn{2}{c}{ Semanas después de la aplicación } \\
\cline { 4 - 5 } & 400 & 96 & $\mathbf{1}$ & $\mathbf{2}$ \\
\hline Icasso 24 EC & 500 & 120 & $68 \mathrm{a}$ & $86 \mathrm{a}$ \\
Icasso 24 EC & 800 & 96 & $63 \mathrm{~b}$ & $91 \mathrm{a}$ \\
Arrow 12 EC & & $93 \mathrm{~b}$ &
\end{tabular}

"A todos los tratamientos se le agregó Basagran $48 \mathrm{SL}$ (Bentazon) a 3.0 L/ha equivalente a 1,440 g de ingrediente activo por hectárea; además se añadió el surfactante Adsee ${ }^{\circledR} 775$ a $0.25 \% \mathrm{v} / \mathrm{v}$.

$\S$ Valores en la columna, seguidos por letras iguales, no son estadísticamente diferentes al $5 \%$.

Cuadro 2. Porcentaje del control de malezas gramíneas con dos formulaciones y dos dosis del herbicida Clethodim?.

\begin{tabular}{lccccc}
\hline $\begin{array}{c}\text { Nombre comercial y } \\
\text { formulación }\end{array}$ & $\begin{array}{c}\text { Producto comercial } \\
(\mathbf{m l} / \mathbf{h a})\end{array}$ & $\begin{array}{c}\text { Ingrediente } \\
\text { activo/ha (g) }\end{array}$ & \multicolumn{3}{c}{ Semanas después de la aplicación } \\
\cline { 4 - 6 } & & 96 & $\mathbf{1}$ & $\mathbf{2}$ & $\mathbf{3}$ \\
\hline Icasso 24 EC & 400 & 120 & $36 \mathrm{~b}$ & $60 \mathrm{a}$ & $40 \mathrm{~b}$ \\
Icasso 24 EC & 500 & 96 & $42 \mathrm{ab}$ & $50 \mathrm{a}$ & $45 \mathrm{~b}$ \\
Arrow 12 EC & 800 & 120 & $37 \mathrm{ab}$ & $49 \mathrm{~b}$ & $33 \mathrm{c}$ \\
Arrow 12 EC & 1000 & $\mathrm{cb}$ &
\end{tabular}

"A todos los tratamientos se le agregó Basagran $48 \mathrm{SL}$ (Bentazon) a $3.0 \mathrm{~L} /$ ha equivalente a 1,440 g de ingrediente activo por hectárea; además se añadió el surfactante Adsee ${ }^{\circledR} 775$ a $0.25 \% \mathrm{v} / \mathrm{v}$.

$\S$ Valores en la columna, seguidos por letras iguales, no son estadísticamente diferentes al $5 \%$.

Evaluación del antagonismo entre Clethodim y Bentazon. En las tres evaluaciones hubo diferencia estadística en el control con las dos formulaciones. También hubo diferencia cuando las formulaciones se aplicaron solas o mezcladas con Bentazon. En ninguna evaluación hubo interacción, lo que indica que los factores (formulación y mezcla de Clethodim con Bentazon) actúan independientes.

En las tres evaluaciones, el control fue mayor con la formulación Icasso 24 EC que con Arrow 12 EC, lo que indica que la formulación Icasso 24 EC es más efectiva que Arrow 12 EC para controlar la maleza Cynodon nlemfuensis (Cuadro 3). El mayor control con Icasso 24 EC se atribuye a los ingredientes inertes ya que se aplicó la misma cantidad del ingrediente activo con las dos formulaciones (120 g de Clethodim). Los ingredientes inertes aumentan la eficacia del herbicida ya que afectan ciertos parámetros como la deposición, retención en la hoja, adherencia, difusión, absorción y translocación en la planta (Culpepper et al. 1999a, Culpepper et al. 1999b, Wanamarta y Penner 1989, Underwood 2000).

Las dos formulaciones de Clethodim sin mezclar con Bentazon tuvieron mayor control que cuando se mezclaron en el tanque con Bentazon (Cuadro 3), lo que indica que hubo antagonismo (Figura 1 y 2) que redujo el control. A la tercera semana después de la aplicación, al mezclar Bentazon con Icasso 24 EC el control se redujo de 74 a $25 \%$, una reducción de $66 \%$. Al mezclar Bentazon con Arrow $12 \mathrm{EC}$ el control se redujo de 60 a 11\%, una reducción de $82 \%$ (Cuadro 3). Hubo mayor antagonismo o reducción del control con la formulación Arrow 12 EC que con Icasso 24 EC. Se determinó que existe antagonismo al mezclar en el tanque Clethodim con Bentazon, en ambas formulaciones hubo antagonismo, pero fue mayor con Arrow 12 EC que con Icasso 24 EC. 
Cuadro 3. Control de la maleza estrella africana (Cynodon nlemfuensis) con dos formulaciones de Clethodim (Icasso 24 EC y Arrow 12 EC) aplicadas solas o mezcladas con Bentazon (Basagran 48 SL) ${ }^{\pi}$

\begin{tabular}{lcccc}
\hline \multirow{2}{*}{ Nombre comercial } & $\begin{array}{c}\text { Ingrediente activo } \\
\mathbf{( g / h a )}\end{array}$ & \multicolumn{3}{c}{$\begin{array}{c}\text { Semanas después de la } \\
\text { aplicación }\end{array}$} \\
\cline { 3 - 5 } & 120 & $61 \mathrm{a}^{\S}$ & $75 \mathrm{a}$ & $74 \mathrm{a}$ \\
\hline Icasso 24 EC & $120+1440$ & $31 \mathrm{c}$ & $27 \mathrm{c}$ & $25 \mathrm{c}$ \\
Icasso 24 EC + Basagran 48 SL & 120 & $54 \mathrm{~b}$ & $61 \mathrm{~b}$ & $60 \mathrm{~b}$ \\
Arrow 12 EC & $120+1440$ & $19 \mathrm{~d}$ & $14 \mathrm{~d}$ & $11 \mathrm{~d}$ \\
Arrow 12 EC + Basagran 48 SL
\end{tabular}

ฯ A todos los tratamientos se le añadió el surfactante Adsee ${ }^{\circledR} 775$ a $0.25 \% \mathrm{v} / \mathrm{v}$.

$\S$ Valores en la columna, seguidos por letras iguales, no son estadísticamente diferentes al $5 \%$.

El antagonismo se ha reportado con el herbicida Sethoxydim (graminicida) al mezclarlo con herbicidas Bentazon o Acifluorfen para el control de hojas anchas (Wanamarta et al. 1989, Holshouser y Coble 1990). También se ha reportado que Bentazon reduce la absorción foliar del Sethoxydim en el control de la maleza gramínea Eleusine indica (Rhodes y Coble 1984, Holshouser y Coble 1990). Debido a que Sethoxydim y Clethodim son herbicidas de la misma familia (Ciclohexanodionas), se espera que tengan un comportamiento similar y por eso se presentó este antagonismo con Clethodim y Bentazon.

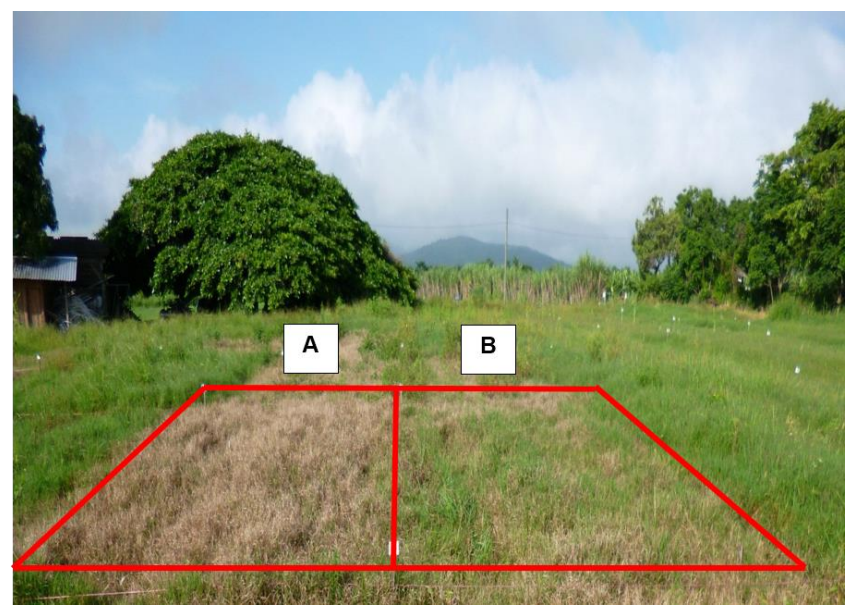

Figura 1. Antagonismo entre Clethodim y Bentazon a los 24 días después de la aplicación. A). Icasso 24 EC aplicado solo a $120 \mathrm{~g}$ de Clethodim. B). Icasso $24 \mathrm{EC}$ aplicado a $120 \mathrm{~g}$ de Clethodim + Basagran 480 SL 3.0 L/ha, control a los 24 días después de la aplicación.
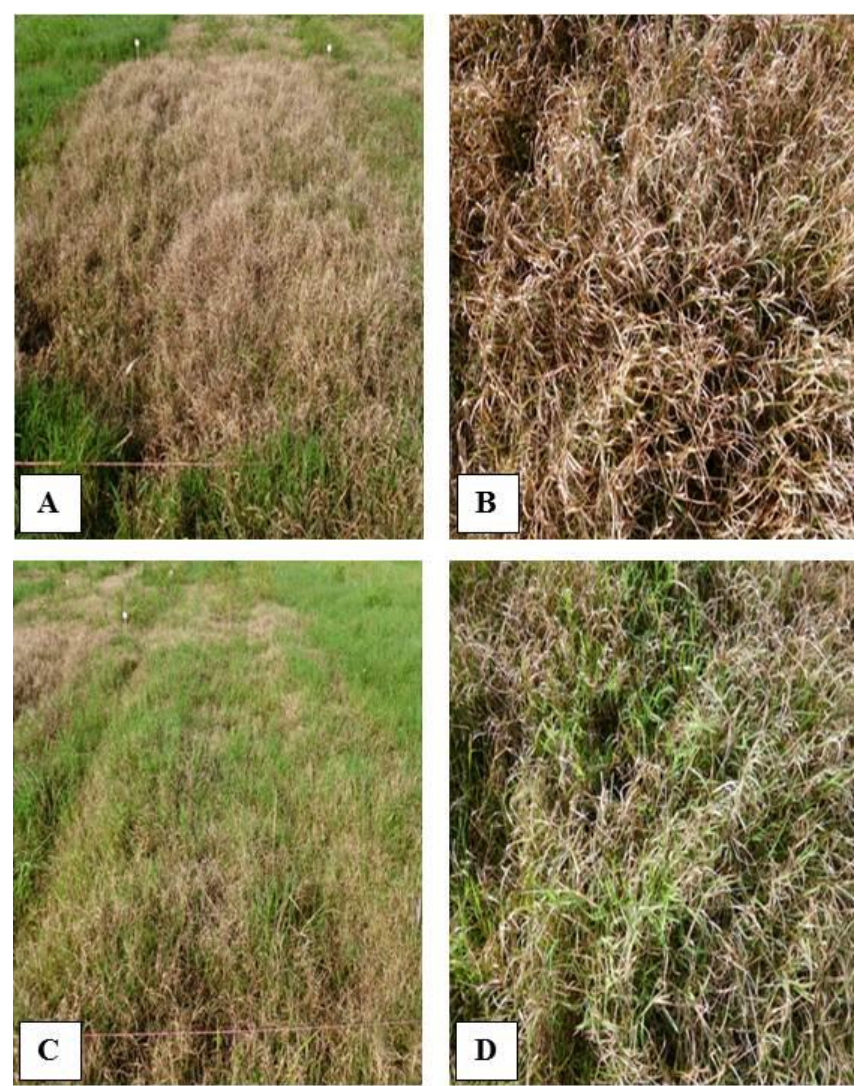

Figura 2. Antagonismo entre Clethodim y Bentazon. Fotos tomadas 24 días después de la aplicación. A). Icasso 24 EC solo, control a los 24 días después de la aplicación. B). Acercamiento del lote aplicado con Icasso 24 EC solo. C). Icasso 24 EC + Basagran 48 SL, control a los 24 días después de la aplicación. D). Acercamiento del lote aplicado Icasso 24 EC + Basagran 48 SL. 
Se ha reportado que al mezclar en el tanque Bentazon con Sethoxydim, la absorción del Sethoxydim por las hojas es menor (Holshouser y Coble 1990), inhibiéndose severamente el efecto del Sethoxydim. Este antagonismo se ha atribuido al sodio que tienes la molécula del Bentazon. Posiblemente lo mismo ocurrió con el Clethodim y Bentazon en este estudio.

\section{Literatura Citada}

Senseman, S.A. 2007. Herbicide Handbook, 7th ed. Champaign, IL: Weed Science Society of America. 458 p.

Australian Weeds Committee. 1979. Guidelines for Field Evaluation of Herbicides. Australian Government Publishing Service. Canberra, Australian. 200 p.

Burke, I.C., J.W. Wilcut. 2003. Physiological basis for antagonism of clethodim by CGA 362622. Weed Science 51:671-677.

Burke, I.C., J.W. Wilcut y D. Porterfield. 2002. CGA 362622 antagonizes annual grass control with clethodim. Weed Technology 16:749-754.

Culpepper, A.S., A.C. York y C. Brownie. 1999a. Influence of bromoxynil on annual grass control by graminicides. Weed Science 47:123-128.

Culpepper, A.S., D.L. Jordan, A.C. York, F.T. Corbin y Y. Sheldon. 1999b. Influence of adjuvants and bromoxynil on absorption of clethodim. Weed Technology 13:536541.

De Prado, R. y M.D. Osuna. 1999. Resistencia a herbicidas. Detección en Campo y Laboratorio. Congreso 1999 de la SEMh. p. 435-440.

Dodds, D.M., D.B. Reynolds, J.H. Massey, M.C. Smith, y C.H. Koger. 2007. Effect of adjuvant and urea ammonium nitrate on Bispyribac efficacy, absorption, and translocation in Barnyardgrass (Echinochloa crusgalli). II. Absorption and Translocation. Weed Science 55(5):406-411.
Godley, J.L. y L.M. Kitchen. 1986. Interaction of Acifluorfen with Fluazifop for Annual Grass Control. Weed Science 34:936-941.

Hatzios, K.K. y D. Penner. 1985. Interactions of herbicides with other agrichemicals in higher plants. Weed Science $1: 1-63$.

Holshouser, D.L. y H.D. Coble. 1990. Compatibility of sethoxydim with five postemergence broadleaf herbicides. Weed Technology 4:128-133.

Kidd, H. y D.R. James. (eds). 1991. The Agrochemicals Handbook, 3era ed. Royal Society of Chemistry Information Services, Cambridge, UK.

Kruse, N.D., R.A. Vidal y M.M. Trezzi. 2006. Curvas de resposta e isobolograma como forma de descrever a associação de herbicidas inibidores do fotossistema II e da síntese de carotenóides. Planta Daninha 24(3): 579_ 587.

Nandula,V.K., D.H. Poston, K.N. Reddy y C.H. Koger,. 2007. Formulation and Adjuvant Effects on Uptake and Translocation of Clethodim in Bermudagrass (Cynodon dactylon L.). Weed Science 55:6-11

Rhodes, G.N., Jr. y H.D. Coble. 1984. Influence of Bentazon on absorption and translocation of sethoxydim in goosegrass (Eleusine indica L.). Weed Science 32:595-597.

Underwood, A.K. 2000. Adjuvant Trends for the New Millennium. Weed Technology 14:765-772

Wanamarta, G. y D. Penner. 1989. Identification of efficacious adjuvants for sethoxydim and bentazon. Weed Technology 3:60-66.

Wanamarta, G., D. Penner y J.J. Kells. 1989. Overcoming antagonistic effects of $\mathrm{Na}$ Bentazon on Sethoxydim absorption. Weed Technology 7:322-325.

WSSA (Weed Science Society of America). 2007. Herbicide Handbook. 9 ed. S.A. Senseman (ed). Laurence, Kansas, Estados Unidos. p 11, 19-21, 132133.

Recibido para publicación el 3 de marzo de 2015. Aceptado para publicación el 13 de julio de 2015. 